\title{
The Enzymatic Basis for the Dehydrogenation of 3-Phenylpropionic Acid: In Vitro Reaction of 3- Phenylpropionyl-CoA with Various Acyl-CoA Dehydrogenases
}

\author{
PIERO RINALDO, JOHN J. O'SHEA, ROY D. WELCH, AND KAY TANAKA \\ Yale University School of Medicine, Department of Human Genetics, New Haven, Connecticut 06510
}

\begin{abstract}
Phenylpropionic acid is an end-product of the bacterial degradation of unabsorbed phenylalanine in the intestinal lumen. As $\mathrm{CoA}$ ester, this metabolite has been considered to be a specific substrate for medium chain acyl-CoA dehydrogenase (MCAD). Its glycine-conjugate, 3-phenylpropionylglycine, has now been established as a pathognomonic marker in urine from patients affected with MCAD deficiency. However, no systematic studies to evaluate the reactivity of 3-phenylpropionyl-CoA with other known acyl-CoA dehydrogenases have so far been carried out to establish the specificity of this substrate for MCAD. We studied the in vitro reactivity of 3-phenylpropionyl$\mathrm{CoA}$ with five rat and human liver acyl-CoA dehydrogenases using purified preparations. We demonstrated that MCAD effectively dehydrogenated 3-phenylpropionyl$\mathrm{CoA}$, and that no other acyl-CoA dehydrogenase exhibited any significant activity with this substrate. In the steady state condition, the $\mathrm{Km}$ of 3-phenylpropionyl- $\mathrm{CoA}$ for human MCAD was $50 \mu \mathrm{M}$. Gas chromatography/mass spectrometry analysis of the assay mixture identified transcinnamoyl-CoA as the product of the reaction. Furthermore, we showed by determination of the reaction products using gas chromatography/mass spectrometry selected ion monitoring that, in absence of the primary electron acceptor, 3-phenylpropionyl-CoA was slowly but significantly dehydrogenated by MCAD under aerobic conditions. These data suggest that MCAD may oxidize 3-phenylpropionyl$\mathrm{CoA}$ in vivo using an alternative electron acceptor, to produce trans-cinnamoyl-CoA. This mechanism provides an explanation for the normal 3-phenylpropionylglycine excretion observed in urine from patients affected with glutaric aciduria type II and ethylmalonic/adipic aciduria. (Pediatr Res 27: 501-507, 1990)
\end{abstract}

\section{Abbreviations}

CI, chemical ionization

CoA, coenzyme A

DCIP, 2,6-dichlorophenol-indophenol

EI, electron impact ionization

ETF, electron transfer flavoprotein

FAD, flavin adenine dinucleotide

GC/MS, gas chromatography/mass spectrometry

IVD, isovaleryl-CoA dehydrogenase

LCAD, long chain acyl-CoA dehydrogenase

MCAD, medium chain acyl-CoA dehydrogenase

Received October 25, 1989; accepted December 19, 1989.

Correspondence and reprint requests: Piero Rinaldo, M.D., Yale University School of Medicine, Department of Human Genetics, 333 Cedar Street, P.O. Box 3333. New Haven, CT 06510-8005.

Supported by Grants DK-29911, DK-17453, and DK-38154 from NIH Grant 1-378 from March of Dimes.
2-meBCAD, 2-methyl branched chain acyl-CoA dehydrogenase

$\mathrm{m} / \mathrm{z}$, mass to charge ratio

PMS, phenazine methosulfate

PPA, 3-phenylpropionic acid

PP-CoA, 3-phenylpropionyl-CoA

PPG, 3-phenylpropionylglycine

SCAD, short chain acyl-CoA dehydrogenase

SIM, selected ion monitoring

TMS, trimethylsilyl (derivative)

Phenylalanine metabolites have been investigated by gas chromatography and GC/MS over the span of more than 20 y (1-3) because of their significance in the biochemical characterization of phenylketonuria and other types of inherited disorders of phenylalanine hydroxylation (4). Among them, PPA is the endproduct of a minor alternative pathway: the bacterial degradation of unabsorbed phenylalanine in the intestinal lumen (5-7). Lactobacillus pastorianus var. quinicus (8), Clostridium difficile (9), and Peptostreptococcus anaerobius (10) represent some of the anaerobic bacterial strains that showed large amounts of PPA in ether-extracts from broth cultures analyzed by GC/MS (11) or liquid chromatography analysis (12). As summarized in Figure 1 , several enzymatic steps are involved in this secondary pathway, where the mechanism of phenyllactic acid transformation to trans-cinnamic acid still requires direct experimental confirmation.

Unlike other phenylalanine metabolites (5-7), PPA is not ordinarily detected as a free acid (13) in either phenylketonuric patients $(1-3)$ or normal subjects, even after a phenylpropionate loading test (14). However, it has been shown that homologous $p$-hydroxy intermediates were produced in tyrosine-loaded rats and rabbits along a parallel pathway (15), suggesting a different endogenous metabolism in other mammalian species. Under normal circumstances, PPA absorbed from the human intestine circulates through the portal system into the liver and diffuses into the hepatocytes, where it is activated to the CoA ester. PP$\mathrm{CoA}$ is transformed to benzoyl-CoA (16), presumably via a $\beta$ oxidation of its aliphatic chain that is initiated by an acyl-CoA dehydrogenase. PP-CoA is then converted to its glycine-conjugate (hippuric acid) by a mitochondrial enzyme, benzoylCoA:glycine $\mathrm{N}$-acyltransferase (17), and finally excreted in urine (7).

The in vivo metabolism of PPA is now receiving considerable attention in a different context in the study of inborn errors of metabolism. Its glycine conjugate, $\mathrm{PPG}$, has proven to be a pathognomonic metabolite for the diagnosis of MCAD deficiency (18). The disease is an autosomal recessive defect of 
mitochondrial $\beta$-oxidation of fatty acids (19). PPG was originally identified by $\mathrm{GC} / \mathrm{MS}$ in urine from a case diagnosed as Reye'slike syndrome (20), and, 2 y later, was detected in urine from eight proven MCAD-deficient patients $(14,21)$. The specificity of PPG for the diagnosis of MCAD deficiency has been recently confirmed by us in $21 \mathrm{MCAD}$-deficient patients including 17 cases who were asymptomatic and untreated at the time of the study (18). The hypothesis that PPA is a product of bacterial metabolism has been corroborated by our observation that, among three diagnostic acylglycines, only PPG excretion was not increased in urine from a MCAD-deficient newborn, in agreement with the gradual bacteria colonization of the intestinal mucosa (Bennett MJ, Coates PM, Hale DE, Millington DS, Pollitt RJ, Rinaldo P, Roe CR, Tanaka K, unpublished data).

In view of these recent development in the study of MCAD deficiency and other inborn metabolic disorders, it is important to ascertain if PP-CoA is metabolized exclusively via MCAD to provide the biochemical basis for the use of PPG as a diagnostic marker for MCAD-deficiency. Previous in vivo (16) and in vitro $(22,23)$ experiments have shown that the shortening of the aliphatic chain of $\omega$-phenyl fatty acids by two carbon atoms is catalyzed by the enzymes of mitochondrial and peroxisomal fatty acid $\beta$-oxidation (23). In an extensive study of the mechanism of the flavin reduction step in the acyl-CoA dehydrogenation pathway, Murfin (22) investigated the dehydrogenation of PP-CoA by mitochondrial octanoyl-CoA dehydrogenase, that was prepared from beef liver. However, it should be noted that there are six known acyl-CoA dehydrogenases $(24,25)$. Three of them, SCAD, MCAD, and LCAD, catalyze the first reaction of the $\beta$ oxidation cycle in the catabolism of fatty acids with varying chain length (26). Two others IVD (27) and 2-meBCAD (28), catalyze the third reaction in the leucine and isoleucine/valine pathways, respectively. Glutaryl-CoA dehydrogenase catalyzes the oxidative decarboxylation of glutaryl-CoA, an intermediate of L-lysine metabolism, yielding as final products crotonyl-CoA and carbon dioxide (29). The first five of these enzymes belong to the acyl-CoA dehydrogenase family (30) and share similar molecular properties and reaction mechanisms (31). All acyl$\mathrm{CoA}$ dehydrogenases remove one hydrogen each from the $\beta$ - and $\alpha$-positions of an acyl-CoA ester. Electrons accepted by the

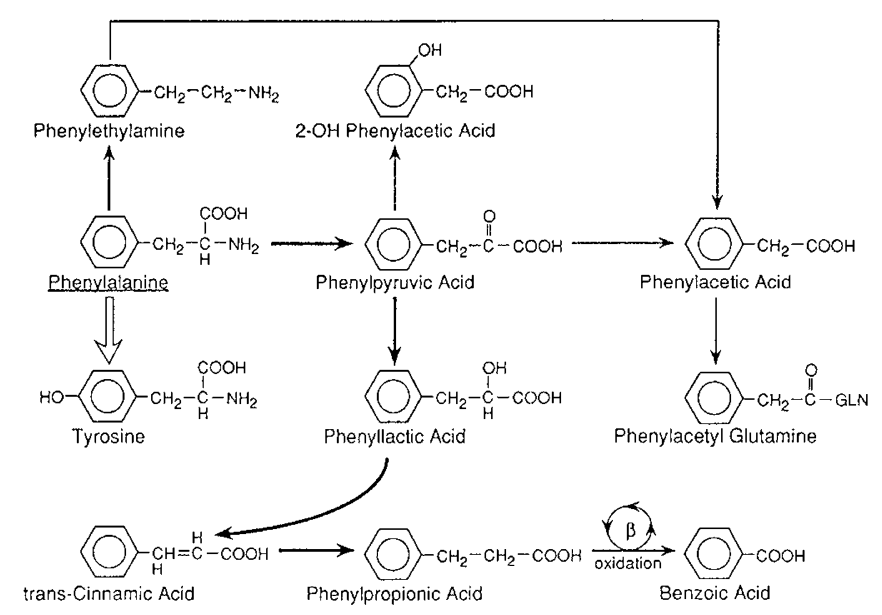

Fig. 1. Phenylalanine metabolism in the intestinal lumen. Proposed scheme for the production of PPA by bacterial degradation of unabsorbed phenylalanine in the intestinal lumen. Wide closed arrows indicate the metabolic pathway from phenylalanine to PPA, narrow arrows indicate additional mechanisms of phenylalanine catabolism that may take place in the intestinal lumen. The wide open arrow shows the conversion to tyrosine, which is the preferential pathway of phenylalanine catabolism. Although tyrosine is not produced from phenylalanine at this level, dietary tyrosine may be metabolized through the same pathway (wide arrows) producing 3-(p-hydroxyphenyl)propionic acid (phloretic acid) (15). enzyme from the substrate are transferred to coenzyme $Q$ in the main mitochondrial electron transport chain via two obligate electron acceptors, ETF and ETF-ubiquinone oxidoreductase (29). It has been observed that the substrate specificities of acylCoA dehydrogenases overlap to some degree (26). So far, no evidence has ever been presented that PP-CoA is dehydrogenated exclusively by MCAD and not by other mitochondrial acyl-CoA dehydrogenases. Thus, the specificity of PPG excretion for the diagnosis of MCAD deficiency has not yet been thoroughly confirmed by excluding other enzyme defects that could impair PPA oxidation and cause PPG accumulation in body fluids.

For these reasons, we synthesized the phenylpropionyl-CoA ester and tested it in vitro as a substrate for five mitochondrial acyl-CoA dehydrogenases purified from rat liver (26-28) and two from human liver (32). Also, a study of the reaction mechanism was carried out by GC/MS analysis of the reaction products. Octanoyl-CoA, the optimal substrate of MCAD, and PP$\mathrm{COA}$ were allowed to react with human MCAD under various assay conditions. These experiments were carried out to confirm the identity of the trans-2-enoyl-CoA products and to explore the mechanism(s) for the normal PPG urinary excretion in patients affected with ETF or ETF-ubiquinone oxidoreductase deficiencies, commonly referred to as glutaric aciduria type II or ethylmalonic/adipic aciduria (29).

\section{MATERIALS AND METHODS}

Materials. CoA lithium salt was obtained from Pharmacia (Milwaukee, WI), FAD and DCIP were purchased from Sigma Chemical Co. (St. Louis, MO), PMS was from Eastman (Rochester, NY). Hydrocinnamic, octanoic, trans-2-octenoic, transcinnamic, and 4-phenylbutyric acids were purchased from Aldrich (Milwaukee, WI). $D_{15}$-Octanoic acid was obtained from Merck, Sharp and Dohme (Montreal, Canada). N,O-bis (trimethylsilyl)-acetamide in pyridine and $1.0 \mathrm{~mL}$ Reactivials were from Pierce Chemical Co. (Rockford, IL). Diazomethane was generated by the method of Schlenk and Gellerman (33) with minor modifications (34).

Synthesis of phenylpropionyl-CoA. PP-CoA was prepared from free $\mathrm{COA}$ and hydrocinnamic acid via the mixed anhydride synthesis $(35,36)$. PP-CoA was purified by ascending paper chromatography using ethanol/0.1 M potassium acetate (1:1), $\mathrm{pH} 4.5$, as the developing solvent. The CoA ester band was visualized under UV-light, cut out, and eluted using distilled water. To remove residual acid, the crude product was acidified to $\mathrm{pH} 1.5$ with $0.1 \mathrm{~N} \mathrm{HCl}$ and extracted six times with equal volumes of ether. The solvent phases were discarded, and the water layer was frozen and lyophilized under vacuum. Yield was checked by hydroxyamate assay (37) and by GC/MS analysis of the acyl moiety as TMS-derivative. All other acyl-CoA substrates were purchased from P-L Biochemicals (Milwaukee, WI) and used without any further purification.

Preparation of enzymes. LCAD, MCAD, SCAD (26), IVD (27), and 2-meBCAD 28 were previously purified to homogeneity from rat liver mitochondria in this laboratory $(30,33)$. Human SCAD and MCAD were purified also to homogeneity from homogenate of autopsy livers (32). The purified enzyme preparations were stored in $50 \%$ glycerol at $-20^{\circ} \mathrm{C}$.

Assay of acyl-CoA dehydrogenase activity. The in vitro assay of acyl-CoA dehydrogenases was carried out spectrophotometrically under aerobic conditions according to the method previously described (26), using PMS and DCIP as the primary electron acceptor and secondary electron acceptor/indicator dye, respectively. The reaction mixture contained $100 \mathrm{mM}$ potassium phosphate buffer (pH 8.0), 3 mM PMS, 0.1 mM FAD, 0.048 $\mathrm{mM}$ DCIP, and $0.1 \mathrm{mM}$ substrate $(0.2 \mathrm{mM}$ in the case of PP$\mathrm{CoA})$. In each control, the optimal substrate for each enzyme (butyryl-CoA for SCAD; octanoyl-CoA for MCAD; palmitoylCoA for LCAD; isovaleryl-CoA for IVD; and isobutyryl-CoA for 2 -meBCAD) was used. The final volume of the reaction mixture 
was $0.5 \mathrm{~mL}$. The amount of enzymes added were: SCAD (rat) $0.05 \mathrm{nmol}$; MCAD (rat), $0.03 \mathrm{nmol}$; LCAD, $0.40 \mathrm{nmol}$; IVD, $0.15 \mathrm{nmol}$; 2 -meBCAD, $0.23 \mathrm{nmol}$; SCAD (human), $0.05 \mathrm{nmol}$, and MCAD (human), $0.08 \mathrm{nmol}$. The enzyme, buffer, and FAD were preincubated at $30^{\circ} \mathrm{C}$ for $5 \mathrm{~min}$ before adding the substrate, DCIP and PMS, the latter as starter of the reaction. The initial rate of DCIP bleaching at $600 \mathrm{~nm}$ wavelength was determined for $30 \mathrm{~s}$ at $30^{\circ} \mathrm{C}$ using a Beckman DU-7 spectrophotometer with a microprocessor (Beckman Instruments, Fullerton, CA).

Identification of the reaction products after incubation of $P P$ CoA and octanoyl-COA with human MCAD. Reaction mixtures were prepared in triplicate as described above, containing 0.08 $\mathrm{nmol}$ of human MCAD and $0.2 \mathrm{mM}$ PP-CoA, and were allowed to react at $37^{\circ} \mathrm{C}$ under aerobic conditions unless otherwise mentioned. One-tenth $\mathrm{mM}$ octanoyl-CoA, the optimal substrate, was separately incubated with MCAD under the same conditions to compare the efficiency of the PP-CoA dehydrogenation reaction. Anaerobic reactions were carried out after repeated cycles of evacuating and purging with argon gas the reaction mixture in a sealed cuvette, followed by a continuous argon flow throughout the incubation period. At the end of the reaction time, the mixtures were transferred to a test tube containing $50 \mu \mathrm{L}$ of 3.5 $\mathrm{MKOH}$ and further incubated at $37^{\circ} \mathrm{C}$ for $30 \mathrm{~min}$ to release the free acid from each acyl-CoA ester. After cooling the mixtures at room temperature, $10 \mu \mathrm{g}$ of 4-phenylbutyric acid (100 $\mu \mathrm{L}$ of 0.1 $\mathrm{mg} / \mathrm{mL}$ solution in methanol) or $50 \mu \mathrm{g}$ of $\mathrm{D}_{15}$-octanoic acid ( 100 $\mu \mathrm{L}$ of $0.5 \mathrm{mg} / \mathrm{mL}$ solution in methanol) were added to the mixture containing the starting substrate PP-CoA or octanoyl$\mathrm{COA}$, respectively. PMS was first removed by extracting the reaction mixture four times with $1.0 \mathrm{~mL}$ of diethyl ether at the basic $\mathrm{pH}$. The samples were then acidified to $\mathrm{pH} 1.0$ with $6 \mathrm{~N}$ $\mathrm{HCI}$ and extracted five times with $1.0 \mathrm{~mL}$ of diethyl ether. The solvent fractions were pooled, dried $1 \mathrm{~h}$ over anhydrous $\mathrm{Na}_{2} \mathrm{SO}_{4}$ and evaporated in a water bath at $37^{\circ} \mathrm{C}$. PPA and trans-cinnamic acids were analyzed as TMS derivatives. They were prepared by heating the evaporated samples in $100 \mu \mathrm{L}$ of TRI-SIL/BSA for $30 \mathrm{~min}$ at $60^{\circ} \mathrm{C}$. The evaporated residues containing octanoate and trans-2-octenoate were redissolved in $100 \mu \mathrm{L}$ of methanol, and methylated by dropwise addition of ethereal diazomethane. After $1 \mathrm{~h}$ at $4^{\circ} \mathrm{C}$, the solvent was evaporated to a final vol of 20 $30 \mu \mathrm{L}$ on an ice-bath under a gentle stream of nitrogen.

A Dani (Finnigan MAT, San Jose, CA) gas chromatograph model 9610 , coupled with a Finnigan model 4510 quadrupole mass spectrometer and a Nova $4 \mathrm{c}$ computer, were used in the SIM mode for the identification and quantitation of the reaction product. Samples were injected in the split mode (ratio 1:20 for total ion current analyses, 1:10 for SIM) into a high-performance fused silica capillary column (Hewlett Packard, Palo Alto, CA): $25 \mathrm{~m}$ length, $0.2 \mathrm{~mm}$ internal diameter, cross-linked $5 \%$ phenyl methyl silicone gum phase, $0.33-\mu$ film thickness. Helium was used as carrier gas at a column flow of $1.0 \mathrm{~mL} / \mathrm{min}$. The injection port and transfer line temperatures were kept at 250 and $275^{\circ} \mathrm{C}$, respectively. The gas chromatographic parameters for the analysis of PPA and trans-cinnamic acid TMS-derivatives, were as follows: starting temperature, $160^{\circ} \mathrm{C}$; initial time, $1.0 \mathrm{~min}$; ramp rate, $8.0^{\circ} \mathrm{C} / \mathrm{min}$; and final temperature, $210^{\circ} \mathrm{C}$ maintained for $0.8 \mathrm{~min}$. The gas chromatographic method for octanoate and trans-2-octenoate methyl-esters used an initial oven temperature of $100^{\circ} \mathrm{C}$, an initial time $0.8 \mathrm{~min}$, a ramp rate of $8.0^{\circ} \mathrm{C} / \mathrm{min}$ and a final temperature of $150^{\circ} \mathrm{C}$. In this case, methylation was preferred over silylation to avoid significant product loss due to the heating of samples during the derivatization procedure.

Calibration curves for GC/MS selected ion monitoring analysis of the substrates and reaction products were prepared by adding increasing amounts of the authentic compound $(5.0$ to $50.0 \mu \mathrm{g})$ to a constant amount of the internal standard in a blank reaction mixture without the enzyme. In the case of PPA and transcinnamic acid, the ratio to the internal standard, 4-phenylbutyric acid, ranged from 0.5 to 2 . For the quantitation of octanoic acid and trans-2-octenoic acid, the ratio to the internal standard, $D_{15}$ octanoic acid, ranged from 0.2 to 1 . The calibration samples were extracted and derivatized through the aforementioned procedures. Linear regression equations gave the following coefficients: PPA, $r=0.9992$; trans-cinnamic acid, $r=0.9997$; octanoic acid, $r=0.9999$; and trans-2-octenoic acid, $r=0.9985$.

First, the identification of the reaction products was verified by the comparison of their EI ionization mass spectra with those obtained by analysis of the respective pure standards. EI mass spectrometric conditions were as used routinely in our laboratory for organic acid analysis (38). Quantitative analysis of the reaction products was then performed under CI conditions in the SIM mode. The mass spectrometer was set up as follows: ion source temperature $130^{\circ} \mathrm{C}$, electron energy $70 \mathrm{eV}$ and emission current $250 \mu \mathrm{A}$. Ammonia was used as the reagent gas at a source pressure held constant at 0.20 torr reading of the pressure gauge. CI mass spectra of the pure standards were recorded by scanning from $\mathrm{m} / \mathrm{z} 100$ to $\mathrm{m} / \mathrm{z} 650$ at a rate of $0.6 \mathrm{scan} / \mathrm{s}$, and suitable ions were chosen for SIM analysis. The dwell time was $100 \mathrm{~ms}$ at each $\mathrm{m} / \mathrm{z}$. The following ions were monitored for analysis of PP-CoA reaction product TMS-derivatives: $\mathrm{m} / \mathrm{z} 240$ (ammonia adduct molecular ion, indicated as $[\mathrm{M}+18]^{+}$) for PPA, $\mathrm{m} / \mathrm{z} 221$ (protonated molecular ion, indicated as $[\mathrm{M}+\mathrm{H}]^{+}$) for transcinnamic acid, and $\mathrm{m} / \mathrm{z} 254\left([\mathrm{M}+18]^{+}\right)$for 4-phenyl butyric acid (internal standard). Methyl-esters of octanoyl-CoA reaction products were analyzed by monitoring $\mathrm{m} / \mathrm{z} 176\left([\mathrm{M}+18]^{+}\right)$for octanoic acid, $\mathrm{m} / \mathrm{z} 174\left([\mathrm{M}+18]^{+}\right)$for trans-2-octenoic acid and $\mathrm{m} / \mathrm{z} 191\left([\mathrm{M}+18]^{+}\right)$for $\mathrm{D}_{15}$-octanoic acid (internal standard). Peak areas were calculated via computer after visual verification of baseline points by the operator.

\section{RESULTS}

The reactivity of PP-CoA was first tested with the preparations of five acyl-CoA dehydrogenases purified from rat liver mitochondria. In the paired experiments, the activity of each enzyme toward the respective optimal substrate was determined and compared to the reactivity of PP-CoA (Table 1). PP-CoA was dehydrogenated at a significant rate by rat MCAD, averaging $48 \%$ of the activity using octanoyl-CoA as substrate under the same conditions. There was no activity when rat SCAD was used. Rat LCAD, IVD, and 2meBCAD exhibited a very low activity with PP-CoA, which represented 1 to $7 \%$ of the activity of each enzyme to dehydrogenate the respective optimal substrate.

In the assays using human SCAD and MCAD, no activity was found with SCAD, but PP-CoA was very efficiently dehydrogenated by human MCAD. The average activity in four experiments was $75 \%$ of that with octanoyl-CoA under the same experimental conditions. In the steady state condition, the Km of PP-CoA for human MCAD was $50 \mu \mathrm{M}$.

Mass spectral identification of the reaction products was achieved by comparison of the mass spectra of the reaction

Table 1. In vitro activities of rat and human acyl-COA dehydrogenases toward 3-phenylpropionyl-CoA*

\begin{tabular}{lcc}
\hline \multirow{2}{*}{ Enzyme } & \multicolumn{2}{c}{ Substrate } \\
\cline { 3 - 3 } & $\begin{array}{c}\text { PP-CoA } \\
(\mu \text { mol DCIP reduced/min/mg protein })\end{array}$ \\
\hline Rat enzymes & & Optimal substrate \\
SCAD & 0.00 & 3.84 \\
MCAD & 7.92 & 15.84 \\
LCAD & 0.07 & 0.96 \\
IVD & 0.03 & 2.43 \\
2meBCAD & 0.04 & 0.66 \\
Human enzymes & & \\
SCAD & 0.00 & 6.14 \\
MCAD & 3.50 & 4.69 \\
\hline
\end{tabular}

* Data represent the mean of assays prepared in triplicate. Zero activity refers to negative values after blank subtraction. 
products, as obtained under EI conditions, with those of the respective authentic standards. Chromatographic retention times and mass spectral fragmentation patterns of the products from PP-CoA and octanoyl-CoA were essentially identical to those for trans-cinnamic ( $\omega$-phenyl-2-propenoic) and trans-2-octenoic acids as TMS-derivatives and methylesters, respectively. Figure 2 shows the mass spectra of trans-cinnamic acid TMS-ester obtained by analysis of the reaction mixture under CI (Fig. 2A) and EI conditions (Fig. $2 B$ ). Here the advantage of using CI for SIM quantitation is apparent by the marked reduction of fragmentation of this compound under $\mathrm{CI}$ conditions and by the prominent intensity of the protonated molecular ion at $\mathrm{m} / \mathrm{z} 221$. These conditions are highly desirable for quantitative analysis through the monitoring of single ionic species (41).

For the quantitative analysis of each compound, ions with the highest relative intensity in the respective $\mathrm{CI}$ mass spectra were selected. They were either the protonated $\left([\mathrm{M}+\mathrm{H}]^{+}\right)$or the $\mathrm{NH}_{4}$ adduct $\left([\mathrm{M}+18]^{+}\right)$molecular ions. Amounts of each compound were calculated by internal standard technique. Table 2 summarizes the results of the reaction product quantitation. In blank reaction mixtures without addition of the enzyme, no signal was detected at the retention time expected for trans-cinnamic or trans-2-octenoic acid under the respective analytical conditions. After incubation for $5 \mathrm{~min}$ in the presence of $0.08 \mathrm{nmol}$ of human MCAD, the dehydrogenation products were readily detectable in the amount that is 38 and $59 \%$ of the sum (in nmol) of the substrate and the respective reaction product for transcinnamoyl-CoA and trans-2-octenoyl-CoA, respectively. The amounts of both products produced after a 10-min incubation were almost identical to those of the 5-min incubation (data not shown).

In the presence of DCIP and the absence of PMS, the primary electron acceptor of the dye reduction assay, no dehydrogenated product was detected from either substrate after $10 \mathrm{~min}$ of incubation. After $30 \mathrm{~min}$ of incubation, however, both products were readily detectable in the enzyme-catalyzed reactions, and the amounts of products further increased two to three times after a 90-min incubation, with the amount of trans-cinnamoylCoA and trans-2-octenoyl-CoA reaching 15.5 and 3.7\%, respectively, of the amount of substrate added (Table 2; Fig. 3). Each product was identified by mass spectrometry as described above. The yield of trans-cinnamoyl-CoA was four times higher than that of trans-2-octenoyl-CoA under identical experimental conditions.

When the reaction was carried out for $90 \mathrm{~min}$ under aerobic conditions with both PMS and DCIP omitted, only a small amount of 2-octenoyl-CoA was produced from octanoyl-CoA. In contrast, $11.3 \%$ of PP-CoA was converted to 2-trans-cinnamoyl-CoA under the same conditions.

\section{DISCUSSION}

In 1908 , in one of the pioneering studies of the $\beta$-oxidation of fatty acids, Dakin (39) demonstrated that PPA fed or injected into a dog was oxidized to benzoic acid and excreted as hippuric acid. More recently, Murfin (22) described that PP-CoA is dehydrogenated by beef liver MCAD. However, no direct evidence has ever been presented that PP-CoA is exclusively dehydrogenated by MCAD and not by other mitochondrial acyl-CoA dehydrogenases. For this reason, we have investigated the in vitro reactivity of $\mathrm{PP}-\mathrm{COA}$ with five purified mitochondrial acyl-CoA dehydrogenases. These enzymes are now recognized as belonging to a gene family (30), sharing major structural and catalytic characteristics, and differing only in the length and configuration of the acyl-CoA substrates. The main aim of our study is to ascertain at the enzymatic level the rationale for using PPG for the biochemical diagnosis of MCAD deficiency, by providing definitive proof that MCAD is responsible for the first step in the PP-CoA degradation.

When PP-CoA was used as substrate, we found no significant activity after incubation with catalytic amounts of LCAD, SCAD, IVD, or 2-meBCAD. In contrast, PP-CoA was dehydrogenated by rat and human MCAD at a rate $48-75 \%$ of that with octanoyl-CoA under the identical experimental conditions. In considering the physiologic significance of these data, the quality of preparations used in this experiment need to be taken into account, because the length of storage and the rate of activity loss during storage are different from enzyme to enzyme. The rat MCAD preparation used here was the latest and best preparation we had, and its specific activity toward octanoyl-CoA had not significantly decreased. The initial specific activities of the rat SCAD, LCAD, IVD, and 2-meBCAD preparations with the respective optimal substrate, measured at the time of purification, were $10.6,2.0,2.7$, and $2.0 \mu \mathrm{mol}$ of DCIP reduced $/ \mathrm{min} /$
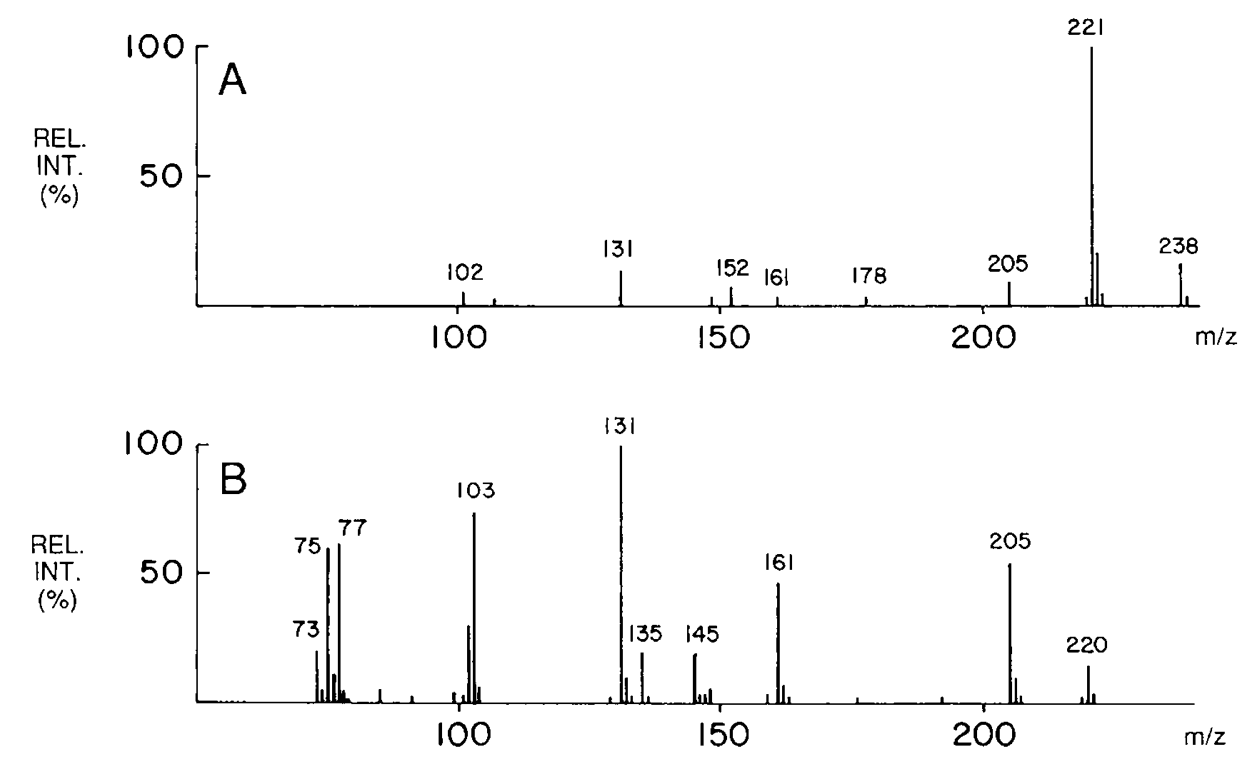

Fig. 2. Demonstration of trans-cinnamic acid as the reaction product. The reaction mixture containing $0.2 \mathrm{mM}$ PP-CoA and a catalytic amount of human MCAD was first hydrolyzed with $3.5 \mathrm{M} \mathrm{KOH}$ for $10 \mathrm{~min}$ at $37^{\circ} \mathrm{C}$. The hydrolyzed mixture was extracted after acidification, and the TMS derivative of the evaporated residue was analyzed by GC/MS. Instrumental conditions are described in Materials and Methods. A. ammonia $\left(0.2\right.$ torr) $\mathrm{CI}$ and $B$, EI ionization mass spectra. In the $\mathrm{CI}$ spectrum, ions at $\mathrm{m} / \mathrm{z} 221$ and $\mathrm{m} / \mathrm{z} 238$ represent the protonated $[\mathrm{M}+\mathrm{H}]^{+}$ and ammonia-adduct $[M+18]^{+}$molecular ions, respectively. 
Table 2. GC/MS reaction product analysis after in vitro incubation of PP-COA and octanoyl-COA with human medium chain acyl-COA dehydrogenase*

\begin{tabular}{|c|c|c|c|c|c|}
\hline \multirow{2}{*}{$\begin{array}{c}\text { Enzyme } \\
\text { added } \\
(\mu \mathrm{L})\end{array}$} & \multicolumn{2}{|c|}{$\begin{array}{l}\text { Electron } \\
\text { acceptors }\end{array}$} & \multirow[b]{2}{*}{$\begin{array}{l}\text { Incubation } \\
\text { time (min) }\end{array}$} & \multicolumn{2}{|c|}{ Amount produced } \\
\hline & PMS & DCIP & & $\begin{array}{l}\text { trans- } \\
\text { cinnamic } \\
(\% \text { of }[\text { Substrate }-\end{array}$ & $\begin{array}{c}\text { trans-2- } \\
\text { octenoic } \\
+ \text { Product]) }\end{array}$ \\
\hline 0 & + & + & 0 & 0.0 & 0.0 \\
\hline 5 & + & + & 5 & 39.0 & 59.3 \\
\hline 5 & - & + & 10 & 0.0 & 0.0 \\
\hline 5 & - & + & 90 & 15.4 & 3.7 \\
\hline 5 & - & - & 90 & 11.4 & 0.6 \\
\hline
\end{tabular}

* The reactions producing trans-cinnamoyl-CoA and those producing trans-octanoyl-CoA were separately performed under aerobic conditions. Results from the experiments performed under identical conditions were summarized in the same lines to allow the comparison of the reactivity of the two substrates. The amount of product detected is given as percentage to the sum of the respective substrate and product, both of which were determined by SIM analysis of the respective free acid after alkaline hydrolysis and ethyl acetate extraction of the mixture.

$\mathrm{mg}$ as measured using the dye-reduction assay (26-28). The activities of the preparations used here were lower than these values as shown in Table 1, indicating unequal loss of activity among them. The rat IVD preparation, like the rat MCAD preparation, had lost little activity. The LCAD preparation has lost approximately $50 \%$ of the original activity, whereas those of the SCAD and 2-meBCAD preparations were 38 and $33 \%$ of the original activity (26-28). The human SCAD and MCAD activities were 84 and $69 \%$ of the respective original activity (32). Even if the loss of the activity LCAD and 2-meBCAD is taken into account, the activities of fresh LCAD and 2-meBCAD with PP-
CoA would be no more than $2 \%$ of that by MCAD. Thus, the data presented in Table 1 clearly establish that MCAD is almost exclusively responsible for the dehydrogenation of PP-CoA in vivo, confirming the specificity and usefulness of urinary PPG determination in the differential diagnosis of MCAD deficiency from other inherited defects of fatty acid metabolism.

PPG excretion has consistently been found not to be increased (18) (Rinaldo P, Tanaka K, unpublished results) in urine from several patients affected with ETF or ETF-ubiquinone oxidoreductase deficiency (29), despite the known requirement of MCAD in vivo for ETF and ETF-ubiquinone oxidoreductase. These findings suggest that an alternative mechanism might be responsible for the in vivo dehydrogenation of $\mathrm{PP}-\mathrm{CoA}$ in a situation where the flow of electrons via ETF and ETF-ubiquinone oxidoreductase to the main mitochondrial electron transport chain is blocked. Our search for the alternative reaction mechanism revealed that in the presence of DCIP alone, omitting PMS, no activity was detectable after 10 min of aerobic incubation with PP-CoA or octanoyl-CoA, but a considerable amount of PP-CoA was dehydrogenated after $90 \mathrm{~min}$. In contrast, the amount of octanoyl-CoA dehydrogenated was only $25 \%$ that of PP-CoA under the same condition. When PMS and DCIP were both omitted, PP-CoA was still dehydrogenated after $90 \mathrm{~min}$ in an amount slightly smaller (75\%) than in the presence of DCIP, whereas the amount of octanoyl-CoA dehydrogenated was negligible. These results indicate that, in the absence of ETF or PMS, PP-COA can be dehydrogenated by MCAD that is reduced by oxygen or other biologic oxidants. The dehydrogenation of PP-COA in the absence of ETF or PMS is not nearly as efficient as in the presence of ETF or PMS, but the rate of this alternative reaction appears to be fast enough to process small amounts of phenylpropionic acid produced in vivo. In this regard, the catalytic behavior of PP-COA is similar to that of $\beta$-(2-furyl)propionyl-CoA, a chromophoric pseudosubstrate with a structure very similar to PP-CoA. McFarland et al. (40) reported the appearance of trans- $\beta$-(2-furyl)acryloyl-CoA in a system
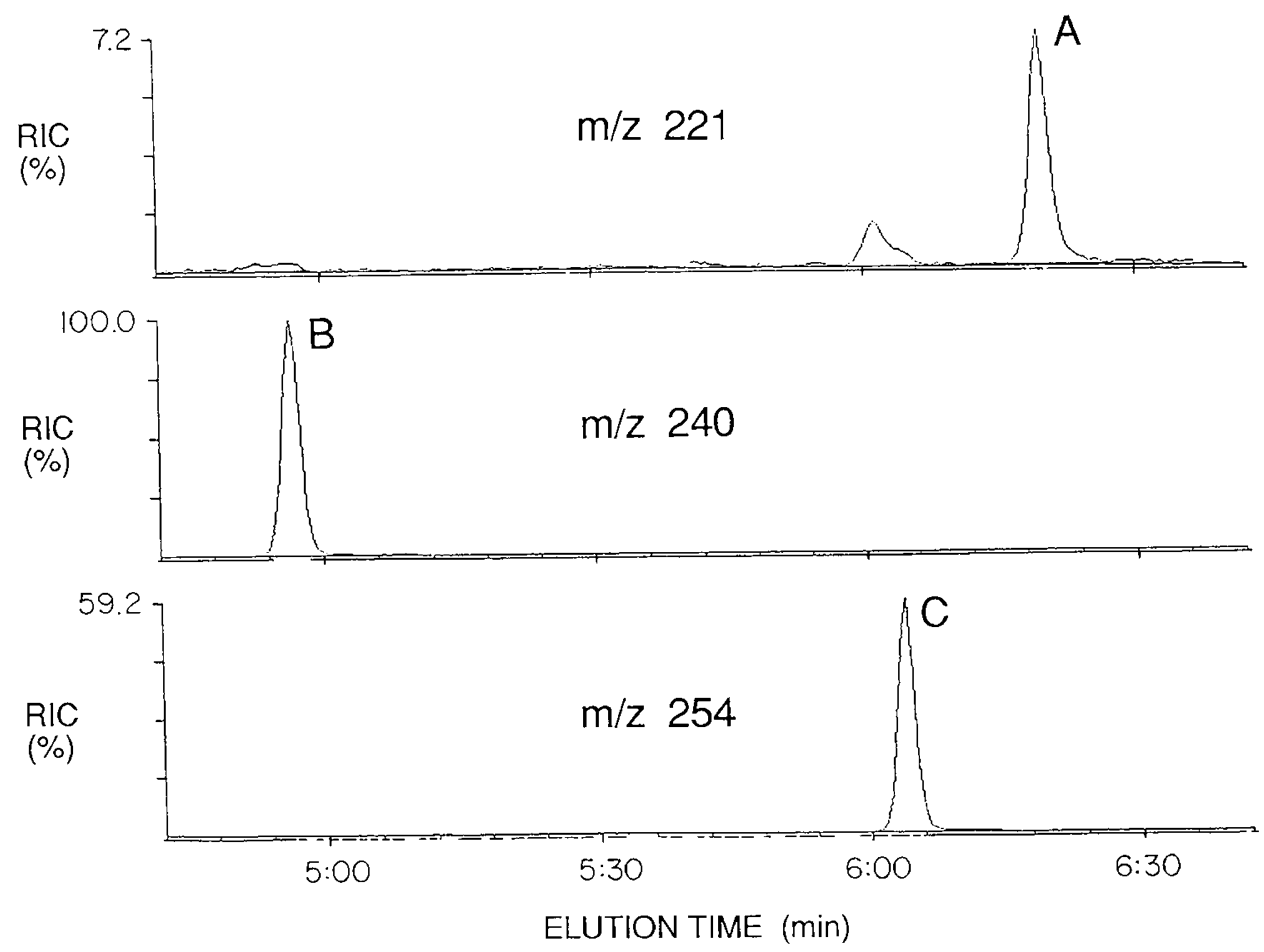

Fig. 3. $\mathrm{Cl}$ (ammonia, 0.2 torr) $[\mathrm{M}+\mathrm{H}]^{+}$ion chromatograms of products from PP-CoA by MCAD in the absence of the PMS. The reaction mixture containing $0.2 \mathrm{mM}$ PP-COA, a catalytic amount of human MCAD and $0.048 \mathrm{mM}$ DCIP was incubated for 90 min under aerobic conditions without the addition of PMS. The reaction mixture was then hydrolyzed, extracted, and derivatized. The mol wt cited below are those of the corresponding TMS-derivatives. Peak legend: $A$, trans-cinnamic acid (mol wt 220, $\left.[\mathrm{M}+\mathrm{H}]^{+}: \mathrm{m} / \mathrm{z} 221\right) ; B, \operatorname{PPA}\left(\mathrm{mol} w \mathrm{wt} 222,[\mathrm{M}+18]^{+}: \mathrm{m} / \mathrm{z} 240\right)$; C, 4-phenylbutyric acid (internal standard, mol wt 236, $[\mathrm{M}+18]^{+}: \mathrm{m} / \mathrm{z} 254$ ). 
where $\beta$-(2-furyl)propionyl-CoA was incubated aerobically with general acyl-CoA dehydrogenase in the absence of electron acceptors. In this condition, oxygen was acting as the electron acceptor resulting in the production of $\mathrm{H}_{2} \mathrm{O}_{2}$. They concluded that "general" acyl-CoA dehydrogenase, as MCAD was referred to in earlier studies, acts as a slow oxidase catalyst producing enoyl-CoA species and $\mathrm{H}_{2} \mathrm{O}_{2}$. Ikeda et al. (41) subsequently pointed out that the oxidase reaction of MCAD with $\beta-(2-$ furyl)propionyl-CoA is likely due to its unusual structure. Upon dehydrogenation at C-2 and C-3, this substrate would have a $\mathrm{C}$ $=\mathrm{C}$ double bond conjugated with an aromatic system and a carboxylate oxygen. Thus, the C-3 hydrogen of this substrate is highly activated, leading to the complete reduction of the enzyme and dissociation of the reduced enzyme/product complex. In contrast, in the reaction of the natural substrates with acyl-CoA dehydrogenases, only the substrate leaves the enzyme/substrate complex (30). As noted above, PP-CoA shares a similar structure with $\beta$-(2-furyl)propionyl-CoA. This peculiar structure of PPCoA with the activated C-3 hydrogen is likely to represent the basis for its ability to use an alternative electron acceptor in its reaction with MCAD. As McFarland et al. (40) suggested for $\beta$ (2-furyl)propionyl-CoA, nicotinamide adenine dinucleotide, ubiquinone, or oxygen are plausible alternatives for ETF. Based on these results, we propose that relatively small but significant amounts of PP-CoA produced in vivo could be dehydrogenated even in the absence of either of the natural electron acceptors. We found that octanoyl-CoA undergoes the same mechanism to a much smaller extent, but its rate seems inadequate to be of any significance in the flow of fatty substrates through the mitochondrial $\beta$-oxidation pathway.

In the disposal of PP-CoA, we must consider the possible role of the peroxisomal $\beta$-oxidation system. Using a partially purified enzyme preparation and phenyl-lauryl-CoA as a model substrate, Yamada et al. (23) demonstrated that approximately $60 \%$ of the $\beta$-oxidation of this substrate in the rat liver was peroxisomal, increasing to $85 \%$ in clofibrate-treated rats. However, they found only trace amounts of phenyl-butyryl-CoA by GC/MS analysis of chain-shortening products, consistent with the poor ability of the peroxisomal $\beta$-oxidation system to metabolize acyl-CoA moieties with a chain length shorter than $C_{6}(42)$. Therefore, it appears credible that PPA metabolism is mainly a mitochondrial process. This view is well supported by our previous data that PPG excretion is significantly increased in urine from patients with MCAD deficiency (18).

Acknowledgments. Rat and human acyl-CoA dehydrogenases were purified by Drs. Yasuyuki Ikeda and Gaetano Finocchiaro, respectively. We thank Ms. Connie Woznick for preparation of this manuscript.

\section{REFERENCES}

1. Hoffman NE, Gooding KM 1969 Gas chromatography of some urinary acid metabolites related to phenylketonuria. Anal Biochem 31:471-479

2. Blau K 1970 Aromatic acid excretion in phenylketonuria. Analysis of the unconjugated aromatic acids derived from phenylalanine. Clin Chim Acta 27:5-18

3. Chalmers RA, Watts RWE 1974 Quantitative studies on the urinary excretion of unconjugated aromatic acids in phenylketonuria. Clin Chim Acta 55:281 294

4. Scriver CR, Kaufman S, Woo SLC 1989 The hyperphenylalaninemias. In Scriver CR, Beaudet AL, Sly WS, Valle D (eds) The Metabolic Basis of Inherited Disease, 6th ed. McGraw-Hill, New York, pp 495-546

5. Van der Heiden C, Wadman SK, Ketting D, De Bref PK 1971 Urinary and faecal excretion of metabolites of tyrosine and phenylalanine in a patient with cystic fibrosis and severely impaired amino acid absorption. Clin Chim Acta 31:133-14!

6. Van der Heiden C, Wauters EAK, Ketting D, Duran M, Wadman SK 1971 Gas chromatographic analysis of urinary tyrosine and phenylalanine metabolites in patients with gastrointestinal disorders. Clin Chim Acta 34:289-296

7. Armstrong MD, Chao FC, Parker VJ, Wall PE 1955 Endogenous formation of hippuric acid. Proc Soc Exp Biol Med 90:675-679

8. Whiting GC, Carr JG 1959 Metabolism of cinnamic acid and hydroxy- cinnamic acids by Lactobacillus pastorianus var. quinicus. Nature 184:14271428

9. Moss CW, Lambert MA, Goldsmith DJ 1970 Production of hydrocinnamic acid by Clostridia. Appl Microbiol 19:375-378

10. Lambert MA, Moss CW 1980 Production of $p$-hydroxycinnamic acid from tyrosine by Peptostreptococcus anaerobius. J Clin Microbiol 12:291-293

11. Moss CW, Nunez-Montiel OL 1982 Analysis of short-chain acids from bacteria by gas-liquid chromatography with a fused capillary column. J Clin Microbiol 15:308-311

12. Guerrant GO, Lambert MA, Moss CW 1982 Analysis of short-chain acids from anaerobic bacteria by high-performance liquid chromatography. $\mathrm{J} C$ lin Microbiol 16:355-360

13. Pollitt RJ 1974 Phenylpropionic acid in the urine of patients with phenylketonuria and normals. Clin Chim Acta 55:317-322

14. Rumsby G, Seakins JWT, Leonard JV 1986 A simple screening test for medium chain acyl-CoA dehydrogenase deficiency. Lancet 2:467

15. Booth AN, Masri MS, Robbins DJ Emerson OH, Jones FT DeEds F 1960 Urinary phenolic acid metabolites of tyrosine. J Biol Chem 235:2649-2652

16. Raper HS, Wayne EJ 1928 A quantitative study of the oxidation of phenylfatty acids in the animal organism. Biochem J 22:188-197

17. Webster LT, Siddiqui UA, Lucas SV, Strong JM, Mieyal JJ 1976 Identification of separate acyl-CoA:glycine and acyl-CoA:L-acyltransferase activities in mitochondrial fractions from liver of rhesus monkey and man. J Biol Chem 251:3352-3358

18. Rinaldo P, O'Shea JJ, Coates PM, Hale DE, Stanley CA, Tanaka K 1988 Medium chain acyl-CoA dehydrogenase deficiency. Diagnosis by stableisotope dilution measurement of urinary n-hexanoylglycine and 3-phenylpropionylglycine. N Engl J Med 319:1308-1313

19. Coates PM, Hale DE, Stanley CA, Corkey BE, Cortner JA 1985 Genetic deficiency of medium-chain acyl coenzyme A dehydrogenase: studies in cultured skin fibroblasts and peripheral mononuclear leukocytes. Pediat Res 19:671-676

20. Rocchiccioli F, Cartier PH, Bougneres PF 1984 Mass spectrometric identification of abnormal aromatic compounds in the urine of a child with Reye's like syndrome. Biomed Mass Spectrom 11:127--131

21. Duran M, Hofkamp M, Rhead WJ, Saudubray JM, Wadman SK 1986 Sudden child death and 'healthy' affected family members with medium chain acylcoenzyme A dehydrogenase deficiency. Pediatrics 78:1052-1057

22. Murfin WW 1974 Mechanism of the flavin reduction step in acyl coenzymeA dehydrogenases. PhD Thesis, Washington University, St. Louis, MO

23. Yamada $Y$, Ogawa S, Horie S, Watanabe T, Suga $T 1987$ Participation of peroxisomes in the metabolism of xenobiotic acyl compounds: comparison between peroxisomal and mitochondrial $\beta$-oxidation of $\omega$-phenyl fatty acids in rat liver. Biochim Biophys Acta 921:292-301

24. Ikeda Y, Dabrowski C, Tanaka K 1983 Separation and properties of five distinct acyl-CoA dehydrogenases from rat liver mitochondria. J Biol Chem 258:1066-1076

25. Lenich AC, Goodman SI 1986 The purification and characterization of glutaryl-CoA dehydrogenase from porcine and human liver. $\mathrm{J}$ Biol Chem 261:4090-4096

26. Ikeda Y, Okamura-Ikeda K, Tanaka K 1985 Purification and characterization of short-chain, medium-chain, and long-chain acyl-CoA dehydrogenases from rat liver mitochondria. J Biol Chem 260:1311-1325

27. Ikeda Y, Tanaka K 1983 Purification and characterization of isovaleryl coenzyme A dehydrogenase from rat liver mitochondria. J Biol Chem 258:10771085

28. Ikeda Y, Tanaka K 1983 Purification and characterization of 2-methylbranched chain acyl coenzyme A dehydrogenase, an enzyme involved in the isoleucine and valine metabolism, from rat liver mitochondria. J Biol Chem 258:9477-9487

29. Frerman FE, Goodman SI 1989 Glutaric acidemia type II and defects of the mitochondrial respiratory chain. In: Scriver CR, Beaudet AL, Sly WS, Valle $\mathrm{D}$ (eds) The Metabolic Basis of Inherited Disease, VIth ed, McGraw-Hill, New York, pp 915-931

30. Matsubara Y, Indo Y, Naito E, Ozasa H, Glassberg R, Vockley J, Ikeda Y, Kraus J, Tanaka K 1989 Molecular cloning and nucleotide sequence of cDNAs encoding the precursors of rat long chain acyl-coenzyme A, shor chain acyl-coenzyme A, and isovaleryl-coenzyme A dehydrogenases. Sequence homology of four enzymes of the acyl-CoA dehydrogenase family. $J$ Biol Chem 264:16321-16331

31. Ikeda Y, Hine DG, Okamura-Ikeda K, Tanaka K 1985 Mechanism of action of short-chain, medium-chain, and long-chain acyl-CoA dehydrogenases. $\mathbf{J}$ Biol Chem 260:1326-1337

32. Finocchiaro G, Ito M, Tanaka K 1987 Purification and properties of short chain acyl-CoA, medium chain acyl-CoA, and isovaleryl-CoA dehydrogenases from human liver. J Biol Chem 262:7982-7989

33. Schlenk H, Gellerman SH 1960 Esterification of fatty acids with diazomethane on a small scale. Anal Chem 32:1412-1414

34. Ramsdell HS, Tanaka K 1977 Gas chromatographic studies of twenty metabolically-important acylglycines. Clin Chim Acta 74:109-114

35. Wieland T, Rueff L 1953 Synthese von S- $\beta$-oxybutyryl- und S-acetyl-Coenzym A. Angew Chem Int Ed Engl 65:186-187

36. Goldman P, Vagalos PR 1961 The specificity of triglyceride synthesis from diglycerides in chicken adipose tissue. J Biol Chem 236:2620-2623

37. Kornberg A, Pricer Jr WE 1953 Enzymatic synthesis of the coenzyme A derivatives of long chain fatty acids. J Biol Chem 204:329-343 
38. Rinaldo P O'Shea JJ, Welch RD, Tanaka K 1989 Stable isotope dilution analysis of $\mathrm{n}$-hexanoylglycine, 3-phenylpropionylglycine and suberyl glycine in human urine using chemical ionization gas chromatography/mass spectrometry selected ion monitoring. Biomed Environ Mass Spectrom 18:471477

39. Dakin HH 1908 Comparative studies of the mode of oxidation of phenyl derivatives of fatty acids by the animal organism and by hydrogen peroxide. J Biol Chem 4:419-435
40. McFarland JT, Lee M-Y, Reinsch J, Raven W 1982 Reactions of $\beta$-(2furyl)propionyl coenzyme A with "general" fatty acyl-CoA dehydrogenase. Biochemistry $21 \cdot 1224-1229$

41. Ikeda Y Okamura-Ikeda K Tanaka K 1985 Spectroscopic analysis of the interaction of rat liver short-chain, medium-chain, and long-chain acyl coenzyme A dehydrogenase with acyl coenzyme A substrates. Biochemistry 24:7192-7199

42. Lazarow PB 1987 The role of peroxisomes in mammalian cellular metabolism. J Inherited Metab Dis 10(suppl 1):11-22

\section{Erratum}

A line was omitted from the abstract of the article entitled "Deficient herpes simplex virus-induced interferon- $\alpha$ production by blood leukocytes of preterm and term newborn infants" by Britta Cederblad et al. (Pediatr Res 27:7-10, 1990). The printer regrets this error. The following is the complete abstract:

ABSTRACT. The ability of peripheral blood mononuclear cells (PBMC) from newborn infants, gestational age 24-42 wk, to produce interferon- $\alpha($ IFN- $\alpha)$ on the first day after birth was studied in vitro. Human amnion cells (WISH) coated with herpes simplex virus type I and fixed by glutaraldehyde were used as IFN- $\alpha$ inducers. Individual IFN- $\alpha$ producing cells (IPC) among PBMC were determined by an immunoplaque assay. The frequency of IPC was low in all premature $(\leq 36 \mathrm{wk})$ infants (median 0.3 IPC $/ 10^{4}$ PBMC, range 0.0-2.6), and significantly higher (median 2.0 IPC/10 ${ }^{4} \mathrm{PBMC}$, range 0.0-16.4) in term infants $(>37 \mathrm{wk})$. The frequencies were lower in both groups of infants than in adults (7.3 IPC $10^{4}$ PBMC, range $\left.2.0-23.7\right)$. When a conditioned medium from cultures of herpes simplex virus type I-stimulated PBMC from adults was added to the IFN induction cultures, the frequencies of IPC increased in PBMC from both preterm and term infants, and in the latter group did not differ significantly from adult levels. The median production of IFN- $\alpha$ per IPC was 1.1 $U$ (range $0.0-2.8$ ) in premature infants, $1.0 \mathrm{U}$ (range 0.0-8.8) in term infants and 3.2 $U$ (range 1.5-8.0) in adults. When concentrations of PBMC in the cultures were decreased, a decline of IPC frequencies occurred. This decline was more marked and started at higher PBMC concentrations in infants than in adults, and was prevented by addition of conditioned medium from herpes simplex virus type I-stimulated cultures of PBMC from adults. The results suggest that PBMC of preterm infants on the first day after birth are deficient both with respect to the proportion of actual IPC and to accessory mechanisms necessary for a normal IFN- $\alpha$ response. In contrast, IPC frequencies in term infants approach levels of adults, but accessory functions may still be deficient. (Pediatr Res 27: 7-10, 1990) 